\title{
Perspectivas ambientales en disputa en el conflicto No Alto Maipo y su comunicación como motivos de oposición al PHAM. Santiago-Chile.
}

\author{
DISPUTED ENVIRONMENTAL PERSPECTIVES IN THE NO ALTO MAIPO CONFLICT \\ AND ITS COMMUNICATION AS REASONS FOR OPPOSITION TO PHAM. SANTIAGO- \\ CHILE
}

Betty Francia*1

bettyfrancia.antropologa@gmail.com

Recibido: 11/7/2020 Aceptado: 09/10/2020

\begin{abstract}
Resumen
Es en el área metropolitana de Santiago de Chile específicamente en el Cajón del Río Maipo el territorio donde se está construyendo el "Proyecto Hidroeléctrico Alto Maipo" (PHAM) desde el año 2008, desde ese mismo año la Coordinadora ciudadana de los Ríos del Maipo (en adelante La coordinadora) lidera un conflicto socioambiental contra ese proyecto, y alerta sobre las amenazas para el ecosistema de la cuenca del Río y la calidad y disponibilidad del agua para Santiago. En el ámbito de la antropología, así como en otras disciplinas, el interés por los modos en que la gente se relaciona con el medio ambiente no es nuevo, desde la etnografía clásica y valiéndose de herramientas para conocer la interacción en las redes sociales virtuales, se analizaron las formas en que el movimiento \#NoAltoMaipo comunicó las amenazas desde su perspectiva ambiental; en cuales y por qué hizo énfasis en las denuncias por la instalación del PHAM. Fue y es éste caso una invitación a la reflexión de las relaciones de los humanos entre sí y con la naturaleza.
\end{abstract}

Palabras clave: ambiente, perspectiva, conflicto, comunicación, Chile.

\begin{abstract}
It is in the metropolitan area of Santiago de Chile, specifically in "Cajón Del Río Maipo", the territory where the "Alto Maipo Hydroelectric Project" (PHAM) has been built since 2008. Since that year the Citizen Coordinator of the Maipo Rivers (hereinafter the coordinator) leads a socio-environmental conflict against this project and warns about the threats to the ecosystem of the River basin and the quality and availability of water for Santiago. In the field of anthropology, as well as in other disciplines, the interest in the ways in which people relate to the environment is not new, from classical ethnography and using tools to know the interaction in virtual social networks, the ways in which the \#NoAltoMaipo movement communicated threats from their environmental perspective were analyzed; in which and why has emphasized complaints about the installation of PHAM. This case was and is an invitation to the reflection of human relationships with each other, but above all with nature.
\end{abstract}

Key words: environment, perspective, conflict, communication, Chile.

\footnotetext{
$1 *$ Universidad de la República.
}

Tekoporá ${ }^{\circledR}$. Centro Universitario de la Región Este. Universidad de la República (C) Francia (2020)

Este es un artículo de Acceso Abierto distribuido bajo licencia Creative Commons (CC BY NC 4.0) 


\section{Introducción}

La comuna de San José de Maipo (llamada comúnmente Cajón del Maipo), se encuentra ubicada al suroriente de la Región Metropolitana, en la zona central de Chile, tiene una superficie de 4.989 kilómetros cuadrados. El Cajón del Maipo, tal como es conocido popularmente, se encuentra a 50 kilómetros al sureste de la ciudad de Santiago, en una zona montañosa de la Cordillera de los Andes.

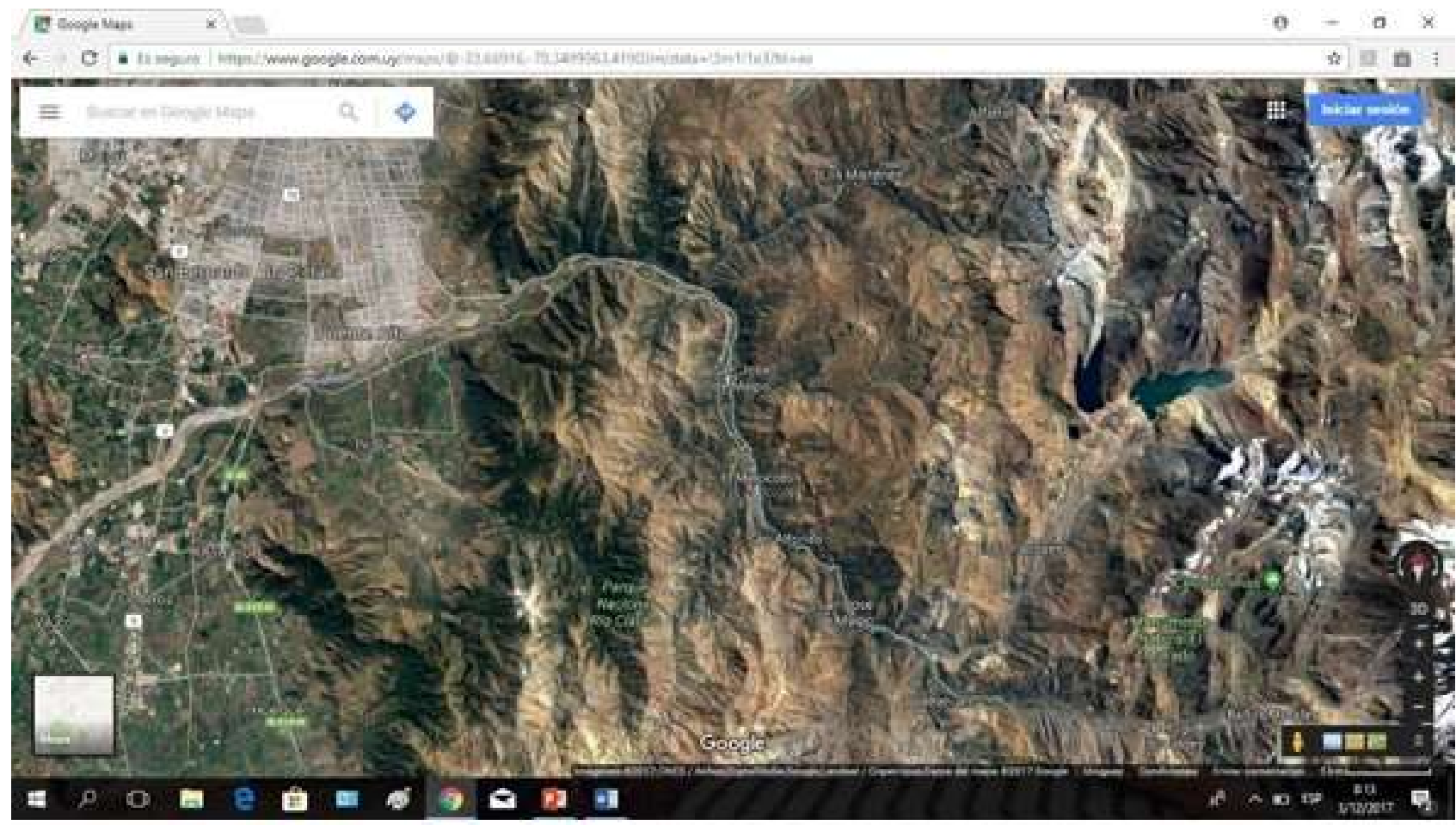

Figura 01. Territorio de la cuenca del río Maipo y del área metropolitana de la ciudad de Santiago de Chile. (Fuente: Google.com.uy/maps)

El PHAM de generación de energía hidroeléctrica, contempla la construcción de dos centrales: Alfalfal II y Las Lajas. Estas centrales se ubicarán en la micro cuenca del río Colorado; implica la construcción de $70 \mathrm{Km}$ de túneles por donde se entubará el agua, lo que impactará directamente a los río Colorado, Yeso y Volcán, y de manera indirecta, al río Maipo. Esta es la diferencia sustancial con una central de paso (definición técnica dada por la empresa responsable del PHAM), ya que el río será entubado y ese impacto se produciría por la disminución del caudal del río entre las localidades de San Gabriel hasta Las Lajas.

En octubre del año 2007 se conforma la agrupación social llamada "Coordinadora Ciudadana de los Ríos del Maipo" (en adelante la Coordinadora), integrada por las siguientes organizaciones: Asociación Gremial de Guías y Operadores Turísticos del Cajón del Maipo, Asociación Gremial de Empresarios Turísticos y Hoteleros del Cajón del Maipo, Cámara de Turismo San José de Maipo, Cámara de Turismo de Pirque, Agrupación Pro Defensa del río Maipo, Sindicatos de Areneros Artesanales, Federación de Trabajadores y Profesionales de 
Aguas Andinas e Instituto del Río Colorado. Esta Asociación se opone a la instalación del PHAM ya que considera que afectará negativamente la calidad de vida y las actividades económicas desarrolladas, especialmente aquellas relacionadas al sector turístico, y la disponibilidad de agua de riego y potable. La Coordinadora se define como una organización que reúne a hombres y mujeres que se relacionan de manera cercana con el Cajón del Maipo asociados desde el año 2007.

En el año 2013 con el comienzo de la obra del PHAM, La Coordinadora buscando mayor visibilidad ante el comienzo de la obra de infraestructura da un giro a la comunicación, intensificando la presencia en las redes virtuales. Las redes sociales se convierten en el medio de comunicación por excelencia para la difusión del impacto que estaba teniendo en la obra en la zona, compartían los avances de las demandas por las irregularidades denunciadas y convocaban a las actividades públicas organizadas por el movimiento. En el año 2015 desde esas mismas redes sociales comienzan a difundirse y a convocar a la adhesión con otros conflictos por el agua en todo Chile, dando cuenta de una problemática nacional.

Entonces, observando el rol de las redes sociales virtuales para el movimiento, y atenta a la participación que como usuaria realizo de estas redes, siempre reconociéndome investigadora en esas interacciones, comienzo a considerar la relevancia que para esta investigación tenían las redes sociales virtuales también como herramienta metodológica, incorporando en el trabajo de campo antropológico, la dimensión virtual.

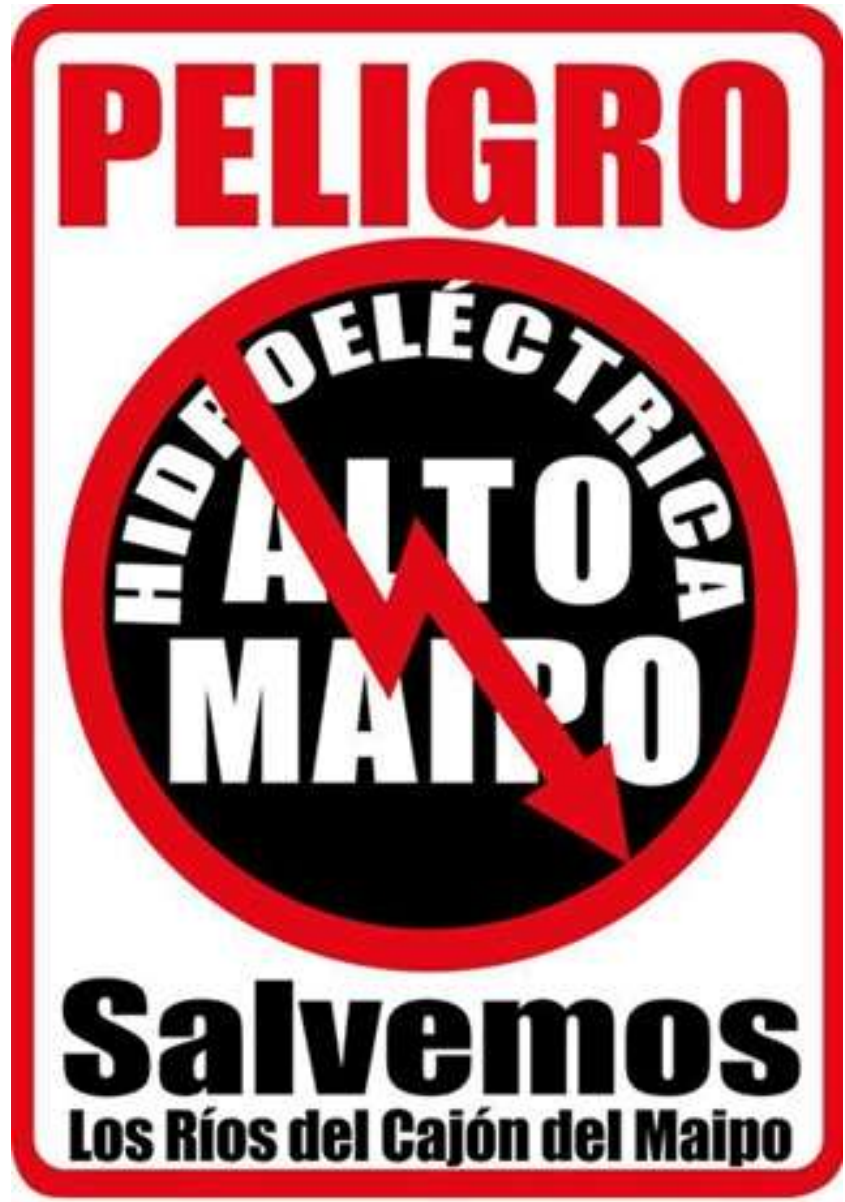

Figura 02. Afiche oficial Movimiento No Alto Maipo

El trabajo de campo en el Maipo se vio caracterizado por tiempos de espera y por la calma característica de zona rural donde los ritmos de la naturaleza acompañan el cotidiano: en el amanecer, comienzo de actividades, pausa para el almuerzo, momentos de reflexión, puesta del sol y cena (Guber, 2001). Los tiempos de espera en el Maipo posibilitaban espacios para recorrer el Cajón, tomar mate, caminar el paisaje, admirar las aguas rápidas del río que más de una vez me detuvo para que simplemente escuchara el sonido profundo de sus aguas, observara su vegetación verde intensa y admirara la neblina constante que durante tres inviernos aprendí a disfrutar. No faltaron las tardes de espera en silencio en la plaza de San José de 
Maipo, lugar de feria de artesanos, viendo pasar trabajadores, estudiantes, mientras llegaba la hora de encuentro con los integrantes de La Coordinadora. En un contexto de conflicto ambiental gran parte de ese tiempo considerado "perdido" fue necesario para que activistas e investigadora lograran construir confianza.

Perspectiva ambiental de los activistas de La Coordinadora en la configuración de los motivos de oposición al PHAM

En relación con los argumentos de oposición, La Coordinadora cuenta con una plataforma de reivindicación donde es evidente que el impacto en la vida cotidiana de los habitantes del Cajón y en el ambiente local, adquieren mayor relevancia.

A su vez, esos motivos forman parte de los comunicados y de las convocatorias a la movilización. A lo largo de los diez años se fueron sumando nuevos motivos ya que inicialmente se centraron en dar a conocer y denunciar la forma de aprobación irregular del PHAM y el impacto ambiental que el proyecto tendría para la zona.

Los activistas de La Coordinadora junto a arrieros, regantes, areneros, sector turístico u hotelero se oponen generando un movimiento que alcanza escalas regionales, nacionales e internacionales. Por lo tanto, la percepción de la naturaleza contribuye a otras dimensiones de la plataforma de reivindicación del movimiento dialogando con sectores claramente productivos, generando una visión común.

La manifestación del problema ambiental como conflicto social, implica un cruce de lógicas, tensiones, donde se compite y se transforma el vínculo entre lo público y lo privado. Los problemas ambientales se presentan como externos, cuando la sociedad los comunica, se constituye como tal, Díaz y Morales, 2010 (citado en Aliste y Urquiza, 2010). Los problemas ambientales son configurados por múltiples miradas, una forma de observarlos es contemplar las distintas lógicas en la que los problemas se desenvuelven.

Utilizando el concepto de percepción para referir a un nuevo estado que el individuo tiene sobre sí mismo y el ambiente en el que vive, da cuenta de una forma de situarnos en el mundo, donde se comprende a las personas y a la naturaleza como inseparables tanto en su definición como en su relación (Descola y Pálsson, 2001). El ambiente pasa a ser un componente activo, la naturaleza se transforma en una fuente de significados, posibilitando estudiar los impactos naturales sobre los grupos humanos (Milton, 2002).

A partir del concepto de Milton "perspectivas culturales", Durand (2000) propone el concepto de: "perspectivas ambientales como el conjunto de normas, supuestos y valores que resultan de la vivencia del entorno natural y permiten comprenderlo y explicarlo." (Durand, 2000, p. 86).

Se presenta así el concepto de "perspectiva ambiental" como nexo entre cultura, percepción e interpretación.

Se parte de una relación entre las perspectivas ambientales y las identidades culturales mucho más compleja, donde en ciertos casos una identidad cultural implica cierto entendimiento particular del mundo pero, en otros, una pluralidad de perspectivas (Milton, 1996 citado en Durand, 2000, p. 86). Siguiendo con esta mirada, se considera que los individuos son los portadores de las perspectivas ambientales y que éstos tienen la posibilidad de moverse 
dentro de un espectro geográfico en el que el contexto social, cultural y económico se transforma:

“el estudio de las perspectivas ambientales puede ayudarnos a explicar cómo algunas personas o grupos sociales perciben y actúan en su ambiente, qué es exactamente lo que aprecian o rechazan y cómo estas experiencias determinan lo que se considera ambientalmente adecuado o permitido." (Durand, 2000, p.87).

Algunas consideraciones sobre el uso de Internet en ciencias sociales

Sin pretensión de ser exhaustivos en la respuesta de las preguntas antes referidas consideramos que el cuasi profético planteo de Arturo Escobar, en "Welcome to Cyberia, notes on the Anthropology of Ciberculture" (2005), nos brinda herramientas heurísticas para abordar el potencial de internet en cuanto a las investigaciones vinculadas al análisis cultural a través de internet. Escobar presenta a la cibercultura vinculada a dos áreas: a) inteligencia artificial, particularmente tecnologías de computación e información y b) biotecnología. Estos temas no son nuevos, es un campo bastamente desarrollado en antropología y la preocupación de muchos por trabajar con una herramienta dinámica y con un potencial innegable. Desde el año 1992 en la reunión anual de la Society for the Social Studies of Science un grupo de antropólogos norteamericanos.

Emerge de ese proceso una nueva interrogante, - ¿estoy haciendo etnografía? Ardèvol, Estalella y Domínguez (2008) en “La mediación tecnológica en la práctica etnográfica”, entiende que:

"los etnógrafos de Internet se han enfrentado a considerables problemas metodológicos en el desarrollo de su trabajo de campo" Utilizamos el concepto "etnografías de Internet" para referirnos a toda una serie de enfoques etnográficos diversos con denominaciones que van desde "etnografía virtual", "etnografía digital", "ciberetnografía”, "etnografía online”, etc." (Ardèvol et al., 2008, p. 10).

La tesis doctoral de la autora, en cuyo marco se dio origen a este artículo, no fue la excepción, exigió repensar la alternancia entre la presencia física y virtual en el campo, online (presencia en las redes) - offline (presencia en el campo) por lo tanto la presencia se hace más frecuente, el campo se amplia y las posibilidades de acceder a la información se multiplican.

"El hecho de considerar la virtualidad como un aspecto más de la vida de los sujetos, permite pensar en la tecnología y en los usos que los sujetos hacen de ella. Y al pensarlo desde esta perspectiva, la construcción de espacios de interacción utilizando Internet se vuelve plausible de ser objeto de análisis 
porque de otro modo, estaríamos frente a un universo ficcional donde nada sería fiable ni auténtico. Situarse frente a la virtualidad como un aspecto más de la vida social del hombre, es lo que posibilita el entrecruzamiento de lo offline y lo online, enriqueciendo las posibilidades de análisis porque de esta manera podemos observar cómo los sujetos construyen prácticas, discursos y recrean experiencias utilizando las nuevas tecnologías". (Capogrossi, Magallanes y Soraire, 2015, p. 54)

Entre los etnógrafos de internet aceptar esto implica rediscutir conceptos guía dentro de la antropología como el de comunidad en este caso la "comunidad virtual", en el marco de ésta investigación no se profundizará en ello debido a que se optó observar la página de Facebook del movimiento y en ella las formas de comunicación en sus convocatorias a eventos. No se abordará el "Grupos público", ni la cuenta de Facebook, lugar donde los usuarios o "amigos" expresan sus motivos personales de oposición al PHAM compartiendo los motivos del arraigo con el Cajón, historias familiares muchas veces íntimas que hacen del Cajón un lugar especial para los activistas, o la oportunidad económica que representa para muchos desarrollar sus emprendimientos destinados a dar servicios al turismo de la zona. En ese grupo sería esperable que tuvieran comportamiento de comunidad virtual, que según expresa Magallanes Udovicich sería:

"un conjunto de personas que se agrupan en el ciberespacio en torno a intereses comunes y que establecen vínculos entre ellos, utilizando como medio de comunicación y relación las herramientas hipermediales que brinda la Internet. En nuestra definición (...) tenemos en cuenta las características que Ontalba (2002) reconoce como habituales en las distintas definiciones de este concepto: a) son agrupaciones de personas, b) reunidas en torno a intereses comunes, y c) que utilizan la Red como medio de relación" (Magallanes, Udovicich, 2010 citado en Capogrossi, Magallanes y Soraire, 2015, p. 9).

Siguiendo a Ardèvol, Estalella y Domínguez (2008) el enfoque metodológico de esta investigación utiliza internet como artefacto, como herramienta para acceder al campo, como forma de seguir el conflicto más allá del tiempo y espacio concreto del trabajo de campo etnográfico, offline.

“(...) lo que proponen algunos investigadores es la realización de una etnografía multisituada que trascienda la necesidad de ubicar el trabajo etnográfico en un territorio delimitado y “(...) permita al etnógrafo seguir a la gente, a los artefactos, a las metáforas, historias o conflictos allí donde se produzcan sin quedar atado a la necesidad de mantener su unidad de análisis en una determinada zona geográfica o en un determinado contexto." (Ardèvol, 2003 citado en Capogrossi et al., 2015, p. 8). 


\section{Estrategia metodológica para el estudio del conflicto}

Las posibilidades tecnológicas con las que hoy podemos contar en el trabajo de campo antropológico sn herramientas valiosas en contextos donde el registro basado en la escritura puede no ser el adecuado; el celular es un dispositivo incorporado al cotidiano de las personas por lo tanto al usar todos el mismo aparato no genera distancia. Como se desarrollará más adelante, las cuestiones éticas sin lugar a dudas con un dispositivo de este tipo, son puestas en juego todo el tiempo ya que las posibilidades que otorga en relación a posibilidad de registro son considerables en cuanto a calidad de imagen y audio. Definir no registrar determinados momentos o pedir autorización en situaciones que el registro podría pasar desapercibido pone en juego los acuerdos previos o requiere de nuevos acuerdos particularmente en un contexto de conflicto socio ambiental como el que se investiga.

Como expresa Izcara (2009), un diseño metodológico cualitativo, se puede ver limitado por el tiempo y por los recursos, pero no puede ser predeterminado a priori ya que el tamaño será óptimo cuando la indagación en el objeto de estudio y objetivos específicos perseguidos queda saturada. De ello dará cuenta la información discursiva recabada, cuando no aparezcan elementos nuevos en relación a los objetivos, "Cuando la producción discursiva total permite interpretar, explicar o describir de forma satisfactoria todas las dimensiones del hecho social objeto de estudio, finaliza el proceso de recopilación de información" (Padget, 1998 citado en Izcara, 2009, p. 104).

Siguiendo la propuesta de George Marcus la investigación se enmarcó en lo que él ha definido como etnografía multisituada o multilocal y la describe de la siguiente forma:

“Otra modalidad de investigación etnográfica, mucho menos común, se incorpora conscientemente en el sistema mundo, asociado actualmente con la ola de capital intelectual denominado posmoderno, y sale de los lugares y situaciones locales de la investigación etnográfica convencional al examinar la circulación de significados, objetos e identidades culturales en un tiempoespacio difuso. Esta clase de investigación define para sí un objeto de estudio que no puede ser abordado etnográficamente si permanece centrado en una sola localidad intensamente investigada. En cambio, desarrolla una estrategia de investigación que reconoce los conceptos teóricos sobre lo macro y las narrativas sobre el sistema mundo pero no depende de ellos para delinear la arquitectura contextual en la que están enmarcados los sujetos. Esta etnografía móvil toma trayectorias inesperadas al seguir formaciones culturales a través y dentro de múltiples sitios de actividad que desestabilizan la distinción, por ejemplo, entre mundo de vida y sistema Holub (1991), distinción a partir de la cual se han concebido múltiples etnografías". (Marcus, 2001, p. 111).

La propuesta metodológica inicial debió ser replanteada luego de cuestionar mi 
participación en las redes sociales. Al interactuar con las cuentas del movimiento generó la necesidad de un profundo replanteo metodológico, entendiendo que tal como explica Marcus "Las etnografías multilocales son inevitablemente el producto de conocimientos de varias intensidades y calidades" (Marcus, 2001, p. 114). La estrategia estaba definida, realizaría una etnografía multilocal, multisituada (Marcus, 2001), realizando trabajo etnográfico clásico y etnografía en y de internet (Marcus, 1996, 2012).

En etnografía en internet se abordaron las redes sociales manejadas por el movimiento como herramienta y fuente, sin interactuar en chats, comentarios, discusiones, pero compartiendo las publicaciones y convocatorias de La Coordinadora en mis redes sociales personales. La etnografía de Internet en el marco de esta investigación refiere al análisis de la comunicación del conflicto en las páginas de Facebook, observación del crecimiento del conflicto por medio de sus cuentas de Twitter y el nexo con otros conflictos por el agua en Chile, el discurso en relación a la convocatoria a eventos y el uso de los videos oficiales de YouTube en entrevistas realizadas a la vocera del movimiento para la reconstrucción de la historia del conflicto.

Las cuentas de Facebook, Twitter y las entrevistas que la vocera concediera a medios de prensa y que se encuentran disponibles en el canal de YouTube de La Coordinadora permiten comenzar a hilar la historia del conflicto, es con ese material analizado que se preparan las entrevistas a los activistas donde se realizan todas aquellas preguntas que permiten entender hechos confusos en la comunicación online.

\section{El campo antropológico, entre el campo online y el offline}

Las estadías en el trabajo de campo fueron asociadas a las actividades más relevantes organizadas por La Coordinadora, como por ejemplo las volanteadas, que consisten en entrega de papel impreso informativo sobre el movimiento No Alto Maipo, que realizan cada domingo con el fin de informar a los visitantes del Cajón sobre el impacto del PHAM. La participación en las actividades se dio por la vía de los hechos, pero también por propia voluntad, estaba allí y entendía que todos los militantes unían sus esfuerzos para dar a conocer el conflicto. Las actividades tenían diversos objetivos por ejemplo desde el celebrar cumpleaños, despedidas, fiestas para recaudar fondos pero también en espacios formales de discusión como la asamblea semanal, marchas y un cabildo auto convocado por parte de los activistas de La Coordinadora.

El nivel de involucramiento en las relaciones que se generó como investigadora con los activistas fue alto. La convivencia, la cotidianeidad con algunas familias y con los demás militantes impulsores del movimiento, todos con larga trayectoria en la defensa por el medio ambiente en Chile, generó un involucramiento mayor una vez explicitado el posicionamiento como investigadora en relación a la temática ambiental. Esto generó empatía y apertura en cuanto a compartir sus objetivos, percepciones y expectativas. El énfasis en el análisis del fenómeno se realizará desde la práctica con La Coordinadora y no desde los actores privados ni estatales, más allá de que ello no se considerara en el proceso de delimitación del tema, se indagó en la información compartida por el PHAM en su web y diarios locales. 
Una de las interrogantes planteadas era: cómo incidía en el trabajo de campo la interacción como usuaria de redes sociales con la cuenta oficial del movimiento por un lado, y por otro con los activistas del movimiento. Algunas de las primeras observaciones se dieron a la llegada al campo en el 2015 cuando fui recibida de forma cordial como si fuéramos viejos conocidos, ya éramos "amigos" en Facebook, algunos activistas me habían empezado a seguir porque veían mi interacción con la cuenta de la coordinadora.

En cuanto a la fiabilidad de la fuente es importante aclarar que los usuarios pueden borrar y editar el posteo que realicen, por ello la definición de trabajar únicamente con el Facebook oficial de La Coordinadora y no con las intervenciones de los activistas, siguiendo específicamente las convocatorias a Eventos, generando descargas de afiches y en caso de justificarse, capturas de pantalla. El supuesto que guía esta decisión es la desaparición de la fuente en 10 años, sumado a la posibilidad de modificar las publicaciones e intervenciones. Por lo tanto fue sistematizada y analizada la comunicación en la cuenta de Facebook oficial del movimiento.

El uso de Facebook como fuente tiene como guía el trabajo realizado por las antropólogas Capogrossi, Magallanes y Soraire (2015) quienes toman como base la propuesta metodológica realizada en la tesis doctoral de Capogrossi (2013a) sobre el caso de los trabajadores de YPF en Campamento Vespucio y General Mosconi, Salta/ Argentina.

Comparto la idea de Sánchez Criado en relación a que: "La tecnología media en la relación de nuestros informantes con el mundo, y el conocimiento sobre el mundo que un antropólogo genera está también mediado por la tecnología que utiliza en su práctica etnográfica. Los artefactos son formas de hacer presente, no sólo de captar una realidad externa; hacen presentes determinadas realidades de una forma determinada, y por lo tanto son actos en los que se niegan u ocultan otras realidades" (Sánchez Criado, 2005, p. 33 citado en Ardèvol, 2008, p. 12). Luego de cada marcha se comparten imágenes y videos por parte de los activistas, esas imágenes muestran lo que quieren compartir seleccionando aquello quieren que los represente, publican en redes sociales y eso les permite revivir y hacer visible lo que defienden.

\section{Uso de las redes sociales virtuales por el movimiento No Alto Maipo.}

En el movimiento No Alto Maipo el activismo se despliega en múltiples ámbitos, las redes sociales juegan un rol fundamental para la llegada del mensaje de forma amplia pero hay un activismo "boca a boca" que realiza cada activista en su cotidiano que es sumamente relevante, activismo como forma de vida, entre sus amigos, espacios de estudio, trabajo.

Los activistas se conectan con los diversos movimientos sociales que reclaman por igualdad y acceso a derechos, quienes militan en \#NoAltoMaipo también lo hacen en otras causas a diferente escala ya sea local o nacional, colaboran entre otras con bibliotecas de la zona realizando trabajo voluntario y a escala nacional con movimientos estudiantiles o sindicatos de trabajadores de diversos sectores. Pero la militancia permea sus cotidianos, es habitual verlos en sus lugares de trabajo o de paseo con remeras con el logo del movimiento y quienes cuentan 
con comercios establecidos colocan pancartas y afiches, saben que ello puede impactar negativamente en su negocio pero aun así definen correr el riesgo.

El movimiento cuenta con una página web oficial cuyo sitio es www.riosdelmaipo.cl con usuario en Twitter \#NoAltoMaipo, Facebook NoAlProyectoAltoMaipo, Instagram y un canal oficial YouTube con 78 videos publicados a mayo del 2017 identificados como "No Alto Maipo". Para el caso de la página de Facebook, La Coordinadora la utiliza con una finalidad concreta de difusión.

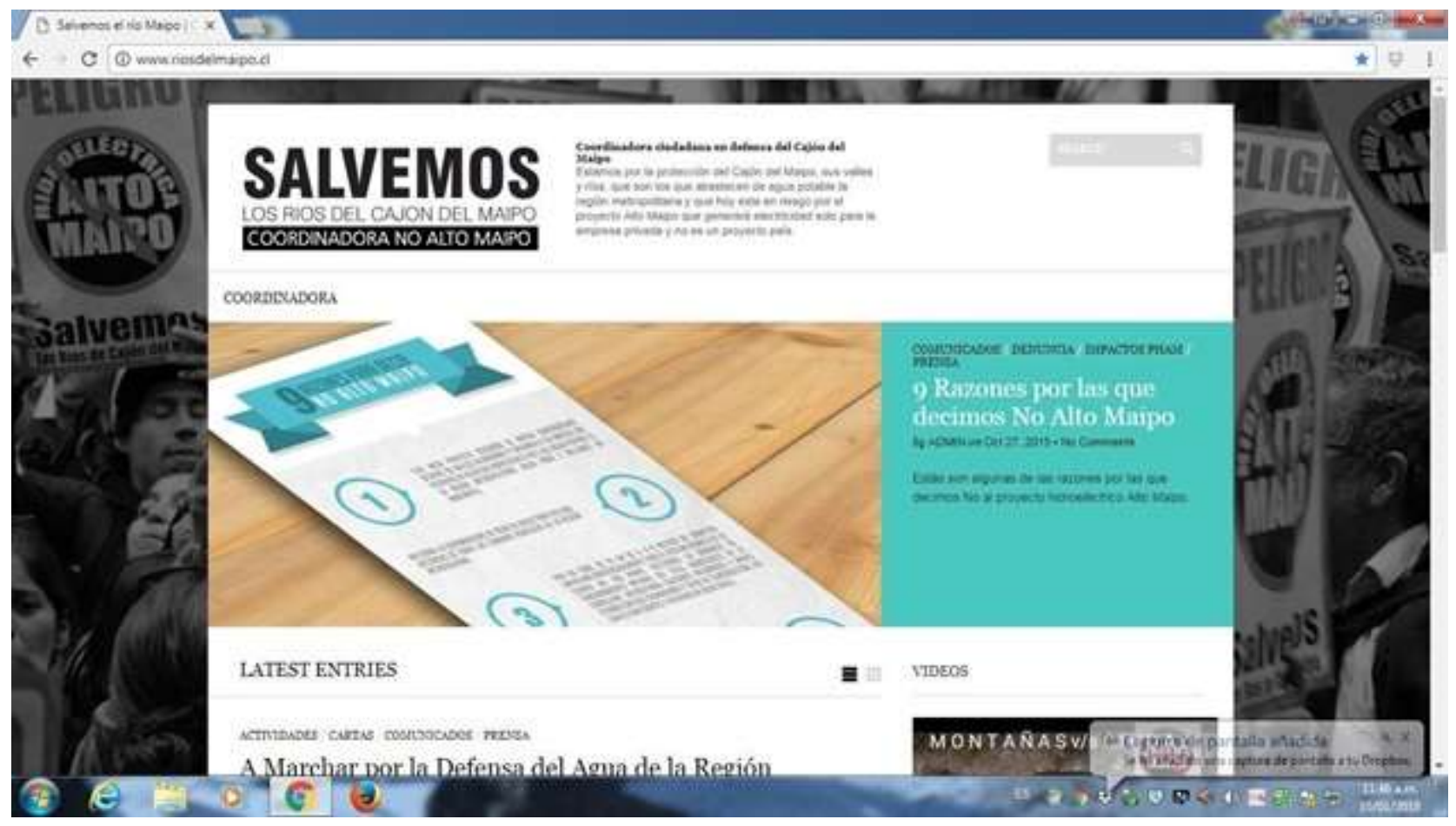

Figura 03- Captura de pantalla web del movimiento No Alto Maipo, con los 9 motivos de oposición al PHAM, base de la plataforma de reivindicación en el conflicto. (Fuente: www.riosdelmaipo.cl)

En las redes sociales se identifica el conflicto No Alto Maipo con el "Hashtag" \#NoAltoMaipo para referirse al mismo. El uso del hashtag tiene por objetivo facilitar la búsqueda de un concepto fácilmente, pero sobre todo, para generar mayor popularidad a lo comunicado y convertirlo en tendencia, a nivel nacional o incluso mundial.

En el siguiente cuadro de elaboración propia fueron identificados los temas comunicados para las convocatorias los diferentes Hashtag usados desde el 2013 al 2017 en las redes sociales virtuales oficiales de La Coordinadora. En el 2017 se incorpora a la comunicación el mensaje y denuncia \#SonTodosCorruptos coincidiendo con noticias públicas de inversiones económicas en el PHAM, de actores vinculados a partidos políticos. 


\begin{tabular}{|c|c|}
\hline $\begin{array}{l}\text { Hashtag } \\
\text { Facebook- Twitter- Instagram }\end{array}$ & \\
\hline 2017 Coordinadora NoAltoMaipo & \\
\hline $\begin{array}{l}\text { \#PorEIAguaDeStgo \#ProtegeEICajón } \\
\text { \#NoAltoMaipo \#SonTodosCorruptos }\end{array}$ & \#NoAltoMaipo \\
\hline 2016 hicieron una actividad y no usaron Hash & ag para convoca \\
\hline 2015 & \\
\hline $\begin{array}{l}\text { \#CajónDelMaipo } \\
\text { \#AltoMaipo } \\
\text { \#ProtegeEICajón \#RíosLibres } \\
\text { \#AltoMaipoMata }\end{array}$ & \#NoAltoMaipo \\
\hline 2014 & \\
\hline $\begin{array}{l}\text { \#DefiendeMaipo } \\
\text { \#MarchaEn2Ruedas } \\
\text { \#UnaSolaFuerza } \\
\text { \#BacheletNoAltoMaipo } \\
\text { \#NOALTOMAIPO }\end{array}$ & \#NoAltoMaipo \\
\hline 2013 & \\
\hline \#NoAltoMaipo & \\
\hline
\end{tabular}




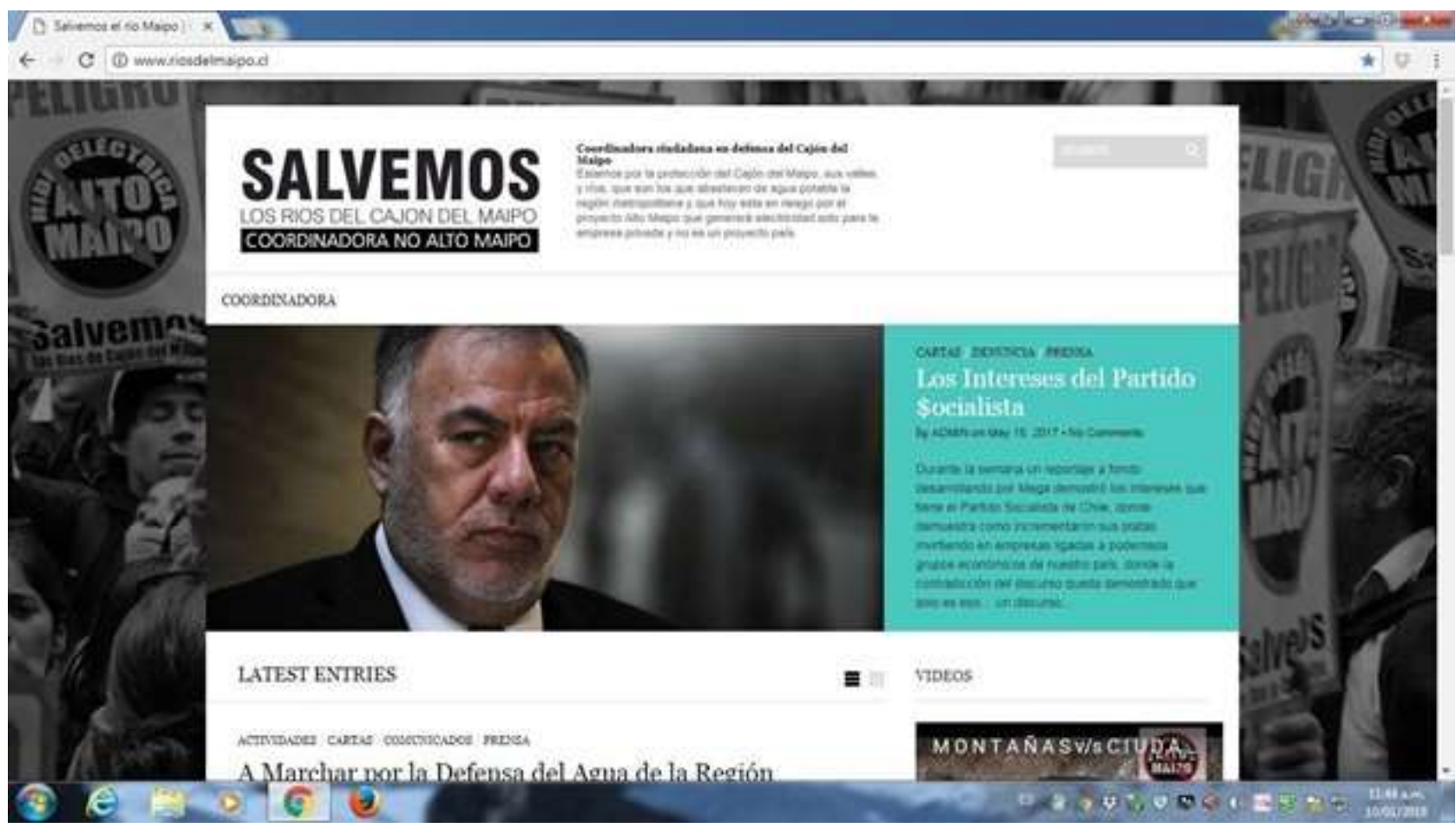

Figura 04 - Captura de pantalla página web oficial del movimiento No Alto Maipo, comunicando en el año 2017 la implicancia del partido socialista (Fuente: www.riosdelmaipo.cl).

Como una forma de visibilizar el conflicto, masificar sus actividades y lograr el aumento de la adhesión a sus demandas, La Coordinadora comienza desde el año 2011 a utilizar las redes sociales como una de sus principales estrategias comunicacionales, especialmente para las personas que no habitan el sector.

\section{Comunicación en la Red social Twitter por parte de la coordinadora}

La cuenta en Twitter Oficial de la Coordinadora Ciudadana de los Ríos del Maipo se identifica con el usuario @NoAltoMaipo. A ese usuario le cambian el nombre en relación con la etapa en la cual se encuentra el proyecto, colocando diferentes tópicos con un \# identificatorio, como por ejemplo \#ChaoAltoMaipo \#NoAltoMaipo \#FueraAltoMaipo. La cuenta fue creada en agosto del 2010 y a octubre del 2017 cuenta con 21.600 seguidores, según terminología twitter $21,6 \mathrm{k}$.

\section{Canal oficial de YouTube}

En el Canal Oficial de YouTube tienen publicados o "colgados" 78 videos subidos a octubre del 2017. En esa red se encuentran subidos por diversos usuarios 22.000 videos vinculados al conflicto en éstos 10 años, YouTube es una plataforma libre que teniendo cuenta 
registrada se pueden compartir videos, los equipos celulares facilitan que en forma instantánea se suban videos desde las actividades y en tiempo real.

\section{Amenazas comunicadas en la red social Facebook}

En Facebook el movimiento cuenta con diversas cuentas con objetivos distintos, crearon una Fan Page Oficial (página de Facebook) donde los "amigos” (así se le llama a los contactos, lo cual no implica que sea un ser humano a quien se le conozca cara a cara o se tenga un vínculo previo ni sostenido de algún tipo) pueden seguir a la cuenta sin esperar la aceptación de ésta para acceder a la información, se comienza a utilizar en el año 2012 el usuario es @NoAlProyectoAltoMaipo y el nombre es “No Alto Maipo”. En el año 2017 llegaron a un total de 190.175 seguidores.

Por otro lado en la plataforma Facebook se puede encontrar otra cuenta con el usuario @noaltomaipo.org nombrada No Alto Maipo, ésta cuenta tiene características que la hacen más compatible con el uso que una persona puede darle a la plataforma Facebook debido a que tiene un número limitado de "amigos" (5.500), el movimiento no actualiza ésta cuenta desde el año 2016 y ya tiene el máximo de "amigos" posibles. Junto a ésta cuenta se encuentra linkeada una página web cuyo sitio es www.noaltomaipo.org; donde se comparten frases de activistas y artistas entre otros.

Las redes sociales son el medio por excelencia donde se convoca a las marchas y el movimiento considera que una marcha multitudinaria invita a otros a pertenecer y a sumarse a la causa. Coincidiendo con los hallazgos de Treré y Cargnelutti (2014) sobre el uso de la red social Facebook por parte del "Movimiento por la paz, justicia y dignidad", los resultados revelan que su uso aparece como un espacio virtual para compartir información y no como un lugar de debate que sería la función principal de una web 2.0. Los espacios de debate se dan en "carne y hueso" (Treré y Cargnelutti, 2014 :198). Metodológicamente estos autores abordan la interacción en las redes y las publicaciones en el estudio del movimiento, analizan la frecuencia de interacción, toman una decisión diferente a la que se toma para la investigación del análisis de las redes del movimiento No Alto Maipo donde se analizan los eventos publicados por el movimiento y la posterior participación virtual en ellos. Por lo que sin antecedentes metodológicos previos concluyo que cada análisis requiere una propuesta de diseño, en ciencias sociales y específicamente en antropología pocos son los aportes al respecto. Entre los más significativos se encuentran los realizados por Árdevol y Lanzani (2014) sobre el uso de las redes sociales por investigaciones sobre la etnografía virtual, "Second life" de Boellstorff (2008) fue enteramente realizada de forma virtual, habla de un "espacio cultural nuevo" uno de los aportes más relevantes que realiza con su investigación es que la distinción entre lo real y lo virtual no está dada de antemano por lo que sostiene que desde la antropología debemos adaptar teoría y metodología para abordar el mundo virtual. Otra aproximación a lo digital son los estudios de los creadores de sistemas y de los hackers (Coleman, 2005).

Analizando los temas de las convocatorias que se realizaban por medio de Facebook. Encontramos que en octubre del 2015 la convocatoria a la marcha nacional marca un hito en la 
forma de incluir a todas las organizaciones sociales y gesta lo que más adelante se conformaría como asamblea permanente.

La comunicación en relación al impacto negativo que la instalación y construcción que el PHAM acarrearía, hasta el 2015 se centraba en temas socioambientales, laborales, fauna, flora, contaminación, algunos ejemplos de esas convocatorias son los siguientes:

- $\quad$ Denunciar actividades laborales y productivas que ya se están dando.

- $\quad$ Riesgos en fauna, flora y la vida del valle.

- $\quad$ Comunican que nunca te vas a enterar por TV cuando gires la llave y no tengas agua, que el tiempo se acaba.

- $\quad$ Cajón del Maipo como zona de sacrificio.

- $\quad$ PHAM contamina.

- $\quad$ El arsénico es el elemento que se reitera a lo largo de todas las convocatorias como el veneno que contamina el agua de Santiago.

- $\quad$ Proyecto inviable ambiental y socialmente.

- $\quad$ Relación megaproyectos y AFPs.

- $\quad$ Defender los bienes naturales.

- $\quad$ Río Maipo como patrimonio de los Chilenos.

- $\quad$ Impacto en la cuenca del Río Maipo y en Santiago.

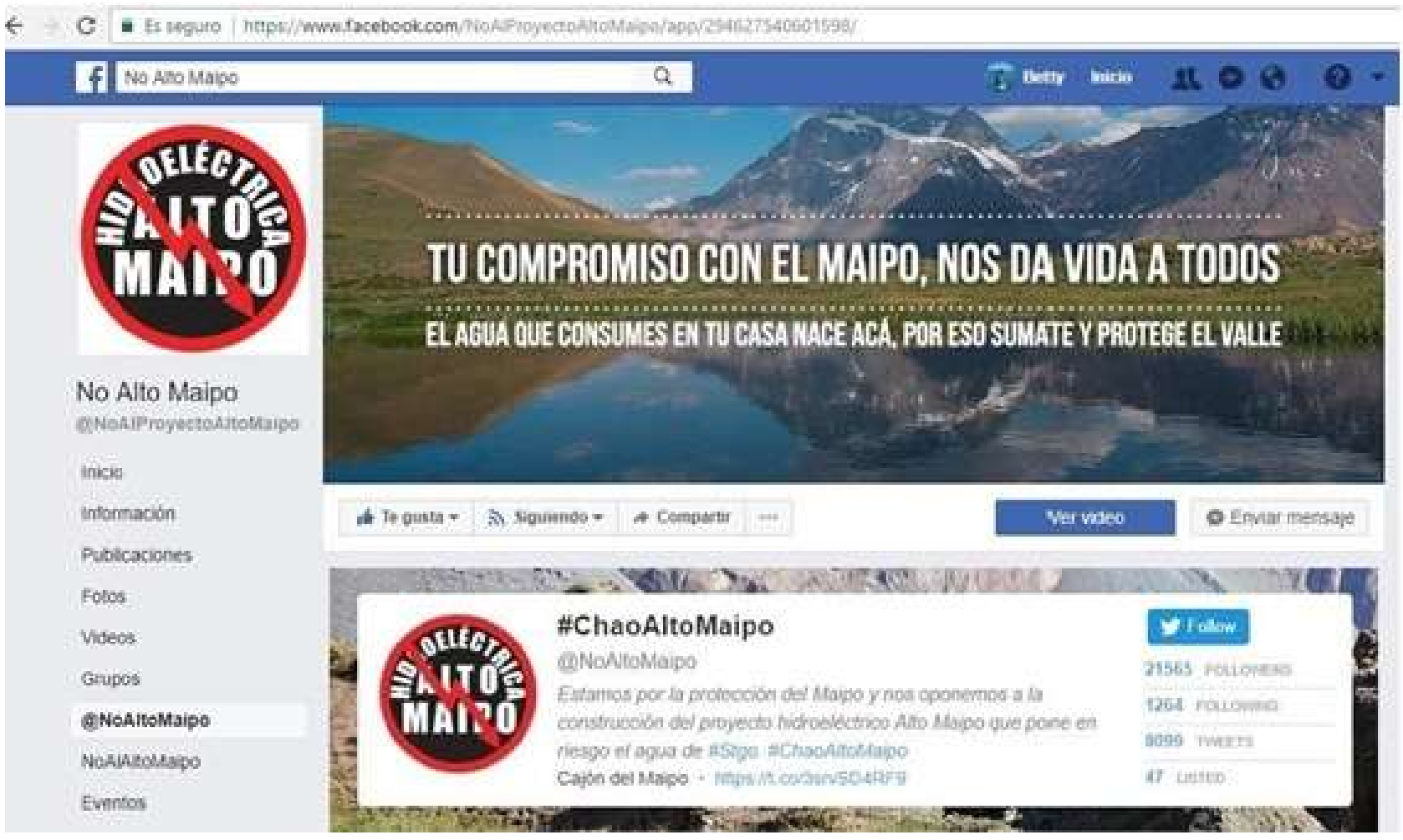

Figura 05- Captura de pantalla de la cuenta oficial de Facebook del movimiento No Alto Maipo (Fuente: Facebook.com/NoAlProyectoAltoMaipo/app/246987654598/) 


\section{Comunicación en el canal oficial de YouTube}

Pero la preocupación y la comunicación de las consecuencias negativas suele centrarse en relación a la escasez del agua de los habitantes del Cajón con sus particularidades de abastecimiento y la incertidumbre sobre el futuro de ese abastecimiento por la falta de estudios oficiales y considerando las afirmaciones que los técnicos vinculados al movimiento alertan.

Las amenazas que difunden en los medios de comunicación son en diversas dimensiones (salud, sociales, políticas) aunque los discursos hacen foco en los daños ambientales también alertan y hacen énfasis sobre las consecuencias en el sector productivo. Se destaca la importancia que tiene como lugar recreativo para la población de Santiago donde la facilidad de acceso lo hace "democrático", pero recobran importancia en cada discurso las consecuencias sociales, sanitarias, la importancia que se le da al Cajón para mantener ese equilibrio natural, esto es comunicado como "vital", al igual que las denuncias vinculadas a corrupción y engaño a la población en la información compartida por AES Gener. Alguna de esas alertas se pueden encontrar en el canal oficial de YouTube del movimiento \#NoAltoMaipo

https://www.youtube.com/user/DefensaRioMaipo

La comunicación de las amenazas socioambientales del PHAM comienzan a incluir en sus temas otras conexiones políticas y económicas, incluso otras causas como por ejemplo otros conflictos como aquellos vinculados a la previsión social, acceso a la vivienda y salud. Esa comunicación deja en evidencia que el conflicto por el agua es un conflicto estructural de inequidad en la sociedad Chilena, víctima de un profundo proceso privatizador de las políticas neoliberales de los años 80.

Los temas adquieren una impronta de denuncia al sistema político, algunos de los mensajes compartidos son los siguientes:

- $\quad$ Agua Andinas cómplice.

- Proyecto calamitoso, financiamiento a políticos, nefasto, injusto, abuso del gobierno, autoridades que no dan respuesta, autoridades cómplices, pago de favores del gobierno con agua, y en el 2013 se centra familia Luksic la imagen del proyecto.

La estrategia de dar cara al proyecto en la imagen de la familia Luksic tendría posteriormente consecuencias que el movimiento valorara como positivas y se atribuyera el triunfo concretamente. Fue la salida del grupo empresarial como accionista mayoritario del proyecto en el año 2017.

\section{Reflexiones finales}

El trabajo de campo se realizó en dos espacios de forma paralela, inicialmente en el espacio virtual online año 2014, utilizando como herramientas las redes sociales virtuales y 
posteriormente desde el año 2015, el espacio offline. Se llevó adelante el trabajo de campo etnográfico en el Cajón del Maipo, lugar donde se conformó La Coordinadora. La guía del mismo, fue conocer los motivos de oposición al PHAM, identificar los medios de comunicación utilizados por el movimiento \#NoAltoMaipo y el rol de las redes sociales.

Una primera reflexión sobre la estrategia metodológica -clave en el desarrollo de la tesisque dio origen a este artículo, es en relación al desafío de plantearse realizar un diseño de sistematización y análisis de las redes sociales utilizadas por los activistas desde y para la antropología. El recorte que se realizó del uso de las redes sociales redujo la complejidad del análisis al máximo, haciendo foco en los "Eventos" convocados por el movimiento en su red social Facebook.

Entonces, por un lado, el abordaje de las redes sociales virtuales genera un insumo metodológico para analizar un problema social donde las mismas son claves como medio de comunicación pero, por otro lado, posibilitó dar respuestas a varios de los objetivos propuestos en la investigación. La etnografía tomada desde su aspecto más tradicional dentro de la antropología permitió comprender y conocer en profundidad los motivos de oposición al PHAM, más allá de lo comunicado en las redes sociales, el discurso de los activistas en el campo offline es sustancialmente más rico y sentido.

Una segunda reflexión es que tanto las comunidades como las organizaciones involucradas en un conflicto estén o no en el territorio donde se instalan los proyectos, cuentan con sus propios esquemas de representación. La percepción de la naturaleza y la historia de la construcción del paisaje dan insumos para ser críticos, entender y oponerse a los efectos de las decisiones que toman otros en sus territorios. Por esto, entender estas percepciones permite entender el impacto social que las comunidades estarían dispuestas a tolerar en sus territorios, desde ahí instalan el conflicto y comunican las amenazas.

Esas percepciones que son a nivel individual y se realizan con los cinco sentidos pasa de ser un acto individual a generar un discurso colectivo entre los activistas del movimiento. Para percibir es indispensable involucrar el cuerpo, estar, exponerse, sentir frío, calor, aromas, sabores, esa condición de involucramiento y acciones en consecuencia es la diferencia sustancial que defienden quienes habitan en el Cajón de forma contrapuesta en sus discursos a los empresarios que actúan movidos por otras emociones y percepciones que no se expresan en la historia o el paisaje del lugar, y que por lo tanto para los activistas carece de validez.

Un arriero, un arenero, un agricultor, involucra su cuerpo y sus emociones en el intercambio productivo con la naturaleza del Cajón, pero su experiencia, la observación de los ciclos naturales le hace saber que necesitan darle a la naturaleza el tiempo necesario para recuperarse.

En el trabajo de campo el tiempo sin tiempo, la pausa, la espera, la contemplación, todo se convertía en instancias de percepción junto a los activistas, los sonidos profundos del Río Maipo, el silencio de una tarde de neblina o un temblor involucraba también mi cuerpo y emociones como investigadora. Entonces se tornó un gran desafío distinguir las percepciones de los activistas, mientras se percibe el mismo sonido del cóndor, la misma brisa o el intenso sonido del río. Las imágenes, los videos ayudan luego a interpretar, a distanciarse, a distinguir 
entre sus percepciones y las propias de la guiada siempre por las preguntas de investigación; pero sigue siendo lo dicho, tanto en entrevistas como en conversaciones casuales lo que le permitió a este escrito distinguir las categorías nativas de percepción de la naturaleza transformadas en plataforma de reivindicación del conflicto con múltiples conexiones a otros temas más allá de lo socioambiental, como lo político, económico y ético.

La percepción y las acciones construyen paisaje y ese paisaje se ve modificado con la instalación del PHAM por "otro", que impone no solo la obra de infraestructura sino también la convivencia con nuevos habitantes y la dinámica de faena, como el aumento del tránsito pesado, el transporte de maquinaria y explosivos por caminos de uso vecinal. Son los activistas quienes alertan a la comunidad sobre esos impactos en sus demandas y manifestaciones públicas.

Con propósitos distintos para las aguas del Río Maipo y diferentes percepciones sobre la naturaleza del Cajón, o la historia vivida del paisaje, las representaciones entran en conflicto, la lucha contra el estado y los megaproyectos que se instalan en las comunidades, generan situaciones crónicas de conflicto territorial. Cierro estas reflexiones dejando abiertas nuevas interrogantes, de qué manera puede construir una historia en paz una sociedad que lleva 13 años viviendo un conflicto cotidiano.

En su escala nacional el movimiento No Alto Maipo generó una importante articulación que posibilitó junto a otros conflictos por el agua o de contenido ambiental situados en otros territorios, construir una plataforma común, teniendo como puntero de lanza la instalación de la asamblea constituyente para la reforma constitucional.

La lucha es no solo por detener el PHAM, por definir las políticas e intervenciones territoriales, junto a otros movimientos la lucha se transforma en la búsqueda de una sociedad más equitativa, "hasta que la dignidad se haga costumbre" como expresa la frase más repetida en las protestas sociales desde el pasado 18 de octubre del 2019. La reivindicación por una asamblea constituyente tiene como fin modificar la constitución aprobada en el año 1980 durante la dictadura militar, posibilitando ceder los derechos de agua a perpetuidad, siendo Chile el único país en el mundo donde el agua se encuentra en manos de privados.

\section{Referencias}

Aliste, E. y Urquiza, A. (Comps.) (2010). Medio ambiente y sociedad: conceptos, metodologías y experiencias desde las ciencias sociales y humanas. Santiago de Chile, Chile: Ril Editores.

Ardèvol, E., Estalella, A. y Domínguez, D. (Coords.) (2008). La mediación tecnológica en la práctica etnográfica. Fotocopias Zorroaga S.L.2008. Comisión de Publicación ANKULEGI.

Ardèvol, E. y Lanzeni, D. (2014). Visualidades y materialidades de lo digital: caminos desde la antropología. Anthropologica, 32(33), pp. 11-38

Boellstorff, Tom (2008). Coming on age in Second Life. Princeton, NJ: Princeton University Press. 
Capogrossi, M.( 2013a), La relación capital-trabajo en economías de enclave y sus transformaciones durante el proceso de ajuste estructural en los 90. El caso de los trabajadores de YPF en Campamento Vespucio y General Mosconi, Salta. Tesis doctoral Doctorado en Antropología, Facultad de Filosofía y Letras, Universidad de Buenos Aires, Inédita.

Capogrossi, M., Magallanes, M. y Soraire, F. (2015). Los desafíos de Facebook. Apuntes para el abordaje de las redes sociales como fuente. Revista de Antropología Experimental (15), Texto 4, pp. 47-63.

Coleman, G. (2005). The social construction of freedom in free and open source software: hackers, ethics, and the liberal tradition. PhD Dissertation, Department of Anthropology, University of Chicago.

Durand, L. (2000). De las percepciones a las perspectivas ambientales. Una reflexión técnica sobre la antropología y la temática ambiental. Revista Nueva Antropología, 21(68). Universidad Autónoma de México: Redalyc.

Escobar, A. (2005). Bienvenidos a Cyberia. Notas para una antropología de la cibercultura. Revista de Estudios Sociales, 22, pp. 15-35.

Guber, R. (2001). La etnografía. Método, campo y reflexividad. Bogotá, Colombia: Grupo Editorial Norma.

Izcara, S. (2009). La praxis de la investigación cualitativa: guía para elaborar tesis. Impreso en México. Fondo Mixto de Fomento a la Investigación Científica y Tecnológica (Fomix)-ConacytGobierno del Estado de Tamaulipas. ISBN: 978-607-402-137-0.

Marcus, G. y Myers, F. eds. (1996). The Traffic in Art and Culture: New Approaches to a Critical Anthropology of Art. University of California Press, Berkeley

Marcus, G. (2001). Etnografía en/del sistema mundo. El surgimiento de la etnografía multilocal. Alteridades, 11(22), pp. 111-127

Marcus, G (2012). Foreword. En Ethnographic and Virtual Words: A hadbook of method. Tom Boellstorff, Bonnie Nardi, Celia Pearce y T.L. Taylor eds. Princeton: Princeton University Press

Milton, K. (2002). Ecologías: antropología, cultura y entorno. Recuperado: 2014, 23 de agosto. Recuperado de: http://www.universidad.edu.uy/retema/files/2013/10/Antropologia_Cultura_Entonno_Milto n_K.pdf 
Treré, E. y Cargnelutti, D. (2014), Movimientos sociales, redes sociales y Web 2.0: el caso del Movimiento por la Paz con Justicia y Dignidad, Communication \& Society / Comunicación y Sociedad, 27(1), pp. 183-203.

Videos de YouTube

Baquedano, C., González, T., Lacomas K., Larraín M, Martínez M., Vivanco, G. (2013, Noviembre 2). Voces por Los Ríos Libres, No Alto Maipo [https://www.youtube.com/watch?v=N3iZIVRPg3c]. Recuperado de: https://www.youtube.com/user/DefensaRioMaipo 